der getroffenen Feststellungen die Annahme eines Schadens an sich rechtlich nicht zu beanstanden, wenn man wie hier mangels gegenteiliger Anhaltspunkte davon ausgeht, dass der Kaufpreis auch den objektiven Wert des Werks (mit lückenloser, zutreffender Provenienz) widerspiegelt.

[25] Doch folgt daraus nicht - wie offenbar das Landgericht annimmt - die völlige Wertlosigkeit eines Kunstwerks, gegen dessen Entstehen zum angegebenen Entstehungszeitpunkt nach sachverständiger Begutachtung Bedenken aus naturwissenschaftlicher Sicht nicht bestehen und für dessen Schaffung durch den entsprechenden Künstler kunsthistorische Expertisen sprechen. In einem solchen Fall (aber auch dann, wenn zwar Zweifel an der Authentizität eines Werks bestehen, aber letztlich nicht feststellbar ist, dass es sich um eine Fälschung handelt, und deshalb zu Gunsten des Angeklagten von einem Originalwerk auszugehen ist) wird auch das ohne oder mit fal- scher Provenienz verschaffte Kunstwerk einen Wert aufweisen, der - bleibt er auch hinter dem gezahlten Kaufpreis zurück - zu einem Schaden, allerdings nur in Höhe der Differenz zwischen Kaufpreis und ermitteltem Wert des Kunstwerks, führt. Da jegliche Erwägungen zum Wert der Originalwerke fehlen, bedarf die Sache insoweit, zweckmäßigerweise unter Heranziehung eines Sachverständigen, neuer Verhandlung und Entscheidung.

[26] 3. Die Entscheidung ist auch insoweit auf den nicht revidierenden Mitangeklagten Z. zu erstrecken (§ 357 StPO).

V.

[27] Die Aufhebung der Strafaussprüche in den Fällen II. 1 und 2 sowie II. 4-6 der Urteilsgründe entzieht dem Gesamtstrafenausspruch die Grundlage. Dies gilt auch hinsichtlich des Mitangeklagten $\mathrm{Z}$..

\title{
Sicherstellung gefälschter Bilder
}

\author{
BayVGH, Beschluss vom 08. Februar 2021 - 10 ZB 20.340
}

\section{Tenor}

I. Der Antrag auf Zulassung der Berufung wird abgelehnt.

II. Der Kläger trägt die Kosten des Zulassungsverfahrens.

III. Der Streitwert für das Zulassungsverfahren wird auf 99.000,- Euro festgesetzt.

\section{Gründe}

I.

[1] Mit seinem Antrag auf Zulassung der Berufung verfolgt der Kläger seine in erster Instanz erfolglose Klage mit dem Antrag, den Bescheid des Bayerischen Landeskriminalamts vom 29. Dezember 2014 aufzuheben und den Beklagten zu verpflichten, dem Kläger alle 440 sichergestellten Gemälde und Grafiken herauszugeben, weiter.

[2] Mit dem streitgegenständlichen Bescheid vom 29. Dezember 2014 wurden die am 15. September 2010 anlässlich der Durchsuchungsbeschlüsse des Amtsgerichts S. vom 18. Juni 2010 und vom 20. August 2010 beschlagnahmten Gemälde und Grafiken (aufgeführt in den Sicherstellungsverzeichnissen vom 15.9.2010) gemäß Art. 25 Nr. 1 PAG (a.F.) sichergestellt und in öffentliche Verwahrung genommen (Nr. 1.) und der Sofortvollzug dieser Sicherstellung und des Verfügungsverbotes (Nr. 2.) gemäß § 80 Abs. 2 Satz 1 Abs. 4 VwGO angeordnet (Nr. 3.). Sämtliche Gemälde/Grafiken seien dem Malstil und Motiven namhafter Künstler nachempfunden und Fälschungen, bei denen die konkrete gegenwärtige Gefahr bestehe, dass sie vom Kläger in betrügerischer Absicht als (angebliche) Originalwerke an Interessenten verkauft würden. Gegen den Kläger würden bereits strafrechtliche Ermittlungsverfahren wegen Veräußerung gefälschter bzw. unechter Gemälde geführt.

[3] Die auf Aufhebung des Sicherstellungsbescheids und Herausgabe der sichergestellten Gemälde und Grafiken gerichtete Klage des Klägers hat das Verwaltungsgericht mit Urteil vom 7. Januar 2020 abgewiesen. Soweit sich das Klagebegehren auf zwei vom Kläger selbst gefertigte Bilder (Asservatennummern 1.25 und 1.26) beziehe, sei die Klage mangels Rechtsschutzbedürfnis unzulässig, weil der Beklagte bereits mehrfach angeboten habe, diese an den Kläger herauszugeben. Im Übrigen sei die Klage unbegründet, weil die im angefochtenen Bescheid vom 29. Dezember 2014 erfolgte Sicherstellung der am 15.9.2010 beschlagnahmten Gemälde/Grafiken rechtmäßig erfolgt sei und den Kläger nicht in seinen Rechten verletze. Der Kläger habe auch keinen Anspruch auf Herausgabe der sichergestellten Gemälde/Grafiken. Die Sicherstellungsverfügung sei inhaltlich hinreichend bestimmt im Sinne des Art. 37 Abs. 1 BayVwVfG. Der Umstand, dass in der Sicherstellungsverfügung auf die Sicherstellungsverzeichnisse vom 15.9.2010 Bezug genommen werde, dem Bescheid aber eine Asservatenliste mit dem Datum „3.12.2013“ beiliege, mache die Anordnung nicht unbestimmt. Denn in dieser Asservatenliste seien die am 15.9.2010 an verschiedenen Stellen beschlagnahmten und deshalb in mehreren Sicherstellungsverzeichnissen aufgeführten Werke insgesamt aufgelistet; das Datum dieser Liste betreffe lediglich den Zeitpunkt ihres Ausdrucks. Für die Beurteilung der Rechtmäßigkeit der Sicherstellungsverfügung komme es auf die Sach- und Rechtslage im Zeitpunkt des Bescheidserlasses an. Aus der maßgeblichen ex ante-Beurteilung habe die Behör- 
de davon ausgehen dürfen, dass es sich bei sämtlichen sichergestellten Bildern um Fälschungen handle und die Sicherstellung der Bilder zur Verhinderung weiterer Straftaten geboten sei. Dem Landeskriminalamt hätten zum maßgeblichen Zeitpunkt verschiedene Gutachten vorgelegen, die belegten, dass es sich bei den untersuchten Bildern (insgesamt über 30) um Fälschungen handle. In dem von der Staatsanwaltschaft S. unter anderem gegen den Kläger wegen Verdachts des gewerbsund bandenmäßigen Betrugs im Zeitraum 2009 bis 15. September 2010 geführten Ermittlungsverfahren (Az. 133 JS 90492/10) sei dem Kläger zur Last gelegt worden, dass er seit mindestens Februar 2009 gefälschte Bilder namhafter Künstler an vier verschiedene Käufer mit großem Gewinn (und einem Schaden von ca. 1,5 Millionen) veräußert habe; er habe diese Fälschungen bei dem Beschuldigten P.K. in Auftrag gegeben, der die Bilder wiederum von der Beschuldigten L.S. anfertigen habe lassen und vor der Weitergabe an den Kläger auf "alt" getrimmt habe. Auch aus dem vom Kläger im Verwaltungsstreitverfahren vorgelegten "positiven Analysen“ ergebe sich jedenfalls nicht, dass es sich bei den betroffenen Bildern um Originale handle. Bei dieser Erkenntnislage habe das bayerische Landeskriminalamt davon ausgehen dürfen, dass es sich bei allen sichergestellten Bildern um Fälschungen handle. Weiter habe auch die Gefahr bestanden, dass die gefälschten Bilder künftig vom Kläger zur Begehung weiterer Straftaten verwendet würden. Es handle sich überwiegend um Werke, die die Urheberschaft namhafter Künstler für sich in Anspruch nähmen. Bei einem Verkauf derartiger Werke sei davon auszugehen, dass sich Käufer finden würden, die bereit seien, hohe Summen zu investieren. Dies zeigten auch die im Strafverfahren gegen den Kläger aufgeführten Geschäfte bzw. Verkaufsbestrebungen (werden im Einzelnen aufgezählt). Der Kläger habe wiederholt versucht, die Bilder als Originale zu verkaufen. Durchgreifende Indizien, die eine andere Einschätzung rechtfertigten, lägen dagegen nicht vor. Demgemäß sei die Sicherstellung und öffentlich-rechtliche Verwahrung ermessensgerecht erfolgt. Der Kläger habe aber auch keinen Anspruch auf Herausgabe der sichergestellten Gemälde/Grafiken. Insoweit sei auf die Sach- und Rechtslage im Zeitpunkt der letzten gerichtlichen Entscheidung abzustellen. Die Voraussetzungen für eine Sicherstellung seien jedoch nicht entfallen. Vielmehr sei weiterhin davon auszugehen, dass die sichergestellten Gemälde/Grafiken - mit Ausnahme der beiden vom Kläger selbst gemalten Bilder - Fälschungen seien. Auch die im Rahmen des verwaltungsgerichtlichen Verfahrens weiter begutachteten Bilder (vier) hätten sich als Fälschungen herausgestellt, weitere seien ohne eingehende Untersuchung von Sachverständigen als Fälschungen beurteilt bzw. Echtheitsbestätigungen abgelehnt worden. Die vorliegenden negativen Gutachten bzw. gutachterlichen Stellungnahmen sowie die Angaben des Klägers, alle Bilder mit Ausnahme von drei Bildern stammten aus der gleichen Quelle, auf die sich die vorliegenden negativen Gutachten bezögen, rechtfertigten die Einschätzung, es handle sich bei den Bildern ausnahmslos um Fälschungen. Denn auch bei den drei Bildern aus anderer Quelle seien Anhaltspunkte für das Vorliegen von Fälschungen gegeben. Bei dieser Indizienlage hätte es dem Kläger oblegen, substantiiert schlüssige Argumente gegen die Bewertung der Bilder als Fälschungen vorzutragen; hieran fehle es. Auch an der Prognose der drohenden Begehung weiterer Straftaten durch den Kläger habe sich nichts geändert. Dieser vertrete nach wie vor die Auffassung, bei den Bildern handle es sich um Originale und ihm sei Unrecht widerfahren. Eine dem Kläger im Strafurteil attestierte Schuldeinsicht und Reue sei in seinem Verhalten nicht erkennbar. Entgegen seinem Vorbringen könnten seine finanziellen Verhältnisse nicht als geordnet bezeichnet werden. Auch diesbezüglich sei die Gefahrenprognosen nicht zu beanstanden. Die vom Kläger ins Spiel gebrachte Kennzeichnung der streitgegenständlichen Bilder als Fälschungen durch optische Aufheller oder fluoreszierende Mittel sei nicht geeignet, einen Betrug und die Täuschung potentieller Erwerber der Bilder wirksam zu verhindern. Schließlich sei auch die Versicherung des Klägers, die Bilder nicht (mehr) als Originale verkaufen zu wollen oder zu können, nicht glaubhaft. Demgemäß teile das Verwaltungsgericht die Befürchtung, der Kläger werde nach einer Rückgabe die Bilder erneut veräußern.

[4] Mit seinem Antrag auf Zulassung der Berufung macht der Kläger ernstliche Zweifel an der Richtigkeit der Entscheidung des Verwaltungsgerichts hinsichtlich der Bestimmtheit der angefochtenen Sicherstellungsverfügung sowie der Sicherstellungsvoraussetzungen nach Art. 25 Nr. 1 PAG a.F., insbesondere der angestellten Gefahrenprognose und der Verhältnismäßigkeit der Maßnahme, sowohl zum Zeitpunkt des Bescheidserlasses als auch der letzten mündlichen Verhandlung des Verwaltungsgerichts, geltend. Ferner weise die Rechtssache sowohl in tatsächlicher als auch in rechtlicher Hinsicht besondere Schwierigkeiten im Sinne von § 124 Abs. 2 Nr. 2 VwGO auf; für Letzteres spreche bereits der umfangreiche Prozessstoff und die lange Prozessdauer sowie die hier besonders schwierige Beurteilung einer gegenwärtigen Gefahr.

[5] Der Beklagte ist dem Zulassungsantrag entgegengetreten und beantragt die Ablehnung des Antrags.

II.

[6] Der auf die Gründe gemäß § 124 Abs. 2 Nr. 1 und 2 VwGO gestützte Zulassungsantrag ist unbegründet. Weder bestehen ernstliche Zweifel an der Richtigkeit der angefochtenen Entscheidung des Verwaltungsgerichts (1.) noch weist die Rechtssache besondere tatsächliche oder rechtliche Schwierigkeiten auf (2.).

[7] 1. Ernstliche Zweifel an der Richtigkeit des angefochtenen Urteils im Sinne des $\S 124$ Abs. 2 Nr. 1 VwGO bestünden dann, wenn der Kläger im Zulassungsverfahren einen einzelnen tragenden Rechtssatz oder eine einzelne erhebliche Tatsachenfeststellung des Erstgerichts mit schlüssigen Gegenargumenten infrage gestellt hätte (BVerfG, B.v. 10.9.2009 - 1 BvR 814/09 - juris Rn. 11; B.v. 9.6.2016 - 1 BvR 2453/12 - juris Rn. 16). Dies ist jedoch nicht der Fall. 
[8] 1.1. Nicht durchgreifend ist zunächst der Einwand, das Erstgericht habe rechtsfehlerhaft angenommen, dass der angefochtene Sicherstellungsbescheid hinreichend bestimmt sei.

[9] Der Kläger rügt, die angefochtene Sicherstellungsverfügung beziehe sich auf „in den Sicherstellungsverzeichnissen vom 15.9.2010" beschlagnahmte Gemälde und Grafiken, wobei unklar sei, auf welche und wie viele Sicherstellungsverzeichnisse konkret Bezug genommen werde. Durch die dem Bescheid beigefügte Asservatenliste werde diese Unklarheit auch in Bezug auf eine etwaige Vollstreckungsmaßnahme nicht beseitigt; vielmehr würden sich Bescheidstenor und die Anlage zum streitgegenständlichen Bescheid nicht nur hinsichtlich der Bezeichnung - "Asservatenliste“ bzw. „Sicherstellungsverzeichnis" -, sondern auch hinsichtlich des Datums - 3.12.2013 statt 15.9.10 - unterscheiden. Für den Betroffenen sei damit nicht klar erkennbar, wie weit der Eingriff in sein Eigentum reichen solle.

[10] Gemäß Art. 37 Abs. 1 BayVwVfG muss der Inhalt der durch Verwaltungsakt getroffenen Regelung eindeutig, klar und vollständig sein. Notwendig ist regelmäßig eine möglichst präzise Bezeichnung von Gegenstand, Art und Umfang der Regelung; ausreichend ist, dass sich der Inhalt durch Auslegung unter Zugrundelegung des Empfängerhorizonts ermitteln lässt (vgl. Müller in Huck/Müller, Verwaltungsverfahrensgesetz, 3. Aufl. $2020 \S 37$ Rn. 9 m. Rsprnachweisen). Der Entscheidungsinhalt muss also so gefasst sein, dass der Adressat ohne weiteres erkennen kann, was genau von ihm gefordert wird bzw. was in der ihn betreffenden Angelegenheit geregelt worden ist (vgl. Tiedemann in BeckOK VwVfG, Bader/Ronellenfitsch, Stand 1.10.2020, § 37 Rn. 19 m.w.N.).

[11] Gemessen daran ist das Verwaltungsgericht zu Recht davon ausgegangen, dass die angefochtene Sicherstellungsverfügung im Sinne des Art. 37 Abs. 1 BayVwVfG hinreichend bestimmt ist. Das Verwaltungsgericht hat zutreffend ausgeführt, dass die insgesamt 440 Gemälde/Grafiken am 15. September 2010 an mehreren Stellen beschlagnahmt worden seien und es deshalb diesbezüglich mehrere Sicherstellungsverzeichnisse gebe. Der Bescheid benennt in seinem regelnden Teil (Tenor) konkret die aufgrund der drei Durchsuchungsbeschlüsse des Amtsgerichts S. vom 18. Juni 2010 (Az.: 1 Gs 344/10, 1 Gs 345/10) und vom 20. August 2010 (Az. 1 Gs 469/10) beschlagnahmten Gemälde und Grafiken, wie sie in den jeweiligen Sicherstellungsverzeichnissen aufgeführt sind. Wie sich aus den Bescheidsgründen zudem eindeutig und unzweifelhaft ergibt, handelt es sich dabei um die bei den auf Anordnung des Amtsgerichts S. erfolgten Durchsuchungen des Anwesens des Klägers (R.-Str. 29 in S.) sowie der Anwesen des anderweitig Verfolgten M.K. (F.-Str. 14 in L. und A.-B.-Str. 53 in M. - „Wohnung K." ) beschlagnahmten insgesamt 440 Gemälde/Grafiken (90 in der Wohnung des Klägers und 350 in der "Wohnung K." in M.). Damit wird der Gegenstand der polizeilichen Sicherstellung sowohl nach seiner Art (Gemälde und Grafiken) wie auch seinem Umfang (konkrete Anzahl der Gemälde und Grafiken) präzise bezeichnet. Zu Recht hat das Verwaltungsgericht weiter ausgeführt, dass der Umstand, dass die Behörde dem Bescheid eine (Asservaten-)Liste mit einer vollständigen und abschließenden Auflistung aller (400) sichergestellten Gemälde und Grafiken beigefügt hat, die das Datum ihrer Erstellung bzw. ihres Ausdrucks „3.12.2013“ aufweist, den Inhalt des Bescheids nicht etwa unbestimmt macht, sondern vielmehr für den Adressaten (Kläger) ohne weiteres erkennbar macht, welche und wie viele seiner Gemälde/Grafiken von der Sicherstellungsmaßnahme betroffen sind.

[12] 1.2. Auch die Rüge, die tatbestandlichen Voraussetzungen der streitgegenständlichen Sicherstellungsanordnung nach Art. 25 Nr. 1 PAG (a.F.) hätten entgegen der Auffassung des Verwaltungsgerichts im Zeitpunkt der Verfügung nicht vorgelegen, greift nicht durch.

[13] Die Polizei kann nach Art. 25 Nr. 1 PAG in der zum Zeitpunkt des Bescheidserlasses gültigen Fassung vom 14. September 1990 (a.F.; nunmehr Art. 25 Abs. 1 Abs. 1 Buchst. a) PAG in der seit 18.5.2018 geltenden Fassung) eine Sache sicherstellen, um eine gegenwärtige Gefahr abzuwehren. Es muss eine konkrete Gefahr für die öffentliche Sicherheit (oder Ordnung) vorliegen. Maßgeblich für die danach anzustellende Gefahrenprognose sind die konkreten Verhältnisse bzw. Gegebenheiten zum Zeitpunkt der angefochtenen Maßnahme (ex-ante Betrachtung); die Annahme einer entsprechenden Gefährdungslage zu diesem Zeitpunkt muss sich auf hinreichende tatsächliche Anhaltspunkte und Erkenntnisse der Behörde stützen können. Auch hier gilt ein mit zunehmendem Ausmaß des möglichen Schadens abgesenkter Grad der Wahrscheinlichkeit des Schadenseintritts (sog. differenzierender Wahrscheinlichkeitsmaßstab; zum Ganzen vgl. Senftl in BeckOK Polizei- und Sicherheitsrecht Bayern, Möstl/Schwabenbauer, Stand 1.11.2020, PAG Art. 25 Rn. 16 ff. m.w.N.).

[14] Gemessen daran hat das Verwaltungsgericht zu Recht festgestellt, dass die die Sicherstellung anordnende Behörde davon ausgehen durfte, dass es sich bei sämtlichen sichergestellten Bildern um Fälschungen handle und die konkrete Gefahr weiterer Straftaten des Klägers durch die Veräußerung bzw. das Inverkehrbringen dieser Fälschungen bestehe. Soweit der Kläger dagegen einwendet, lediglich etwa 10\% dieser Bilder/ Grafiken seien von Gutachtern untersucht und tatsächlich als Fälschungen eingestuft worden, weshalb im Übrigen allenfalls ein Fälschungsverdacht vorgelegen habe, wird dadurch die Gefahrenprognose nicht durchgreifend erschüttert. Denn das Verwaltungsgericht hat ausgehend von den Erkenntnissen im staatsanwaltschaftlichen Ermittlungsverfahren gegen den Kläger wegen des Verdachts des gewerbs- und bandenmäßigen Betrugs durch die Veräußerung gefälschter Bilder namhafter Künstler mit dem zugrundeliegenden "Geschäftsmodell" der Beauftragung der Fälschungen beim (weiteren) Beschuldigten P.K., der seinerseits die Bilder von der (weiteren) Beschuldigten L.S. habe anfertigen lassen und diese dann vor ihrer Weitergabe an den Kläger „auf alt" getrimmt habe, sowie dem Umstand, dass keine der vom Kläger im Verfahren vorgelegten „positiven Analysen" tatsächlich belegt habe, dass es sich bei den 
betroffenen Bildern um Originale handle, in nachvollziehbarer und schlüssiger Weise gefolgert, dass das Landeskriminalamt auf der Basis dieser Erkenntnisse davon ausgehen konnte bzw. musste, dass es sich bei allen sichergestellten Bildern/Grafiken um Fälschungen handle.

[15] Die auf die Annahme (auch) künftig durch den Kläger drohender Straftaten bezogenen Einwände, insoweit seien vom Verwaltungsgericht die im seinerzeit noch laufenden Strafverfahren geltende Unschuldsvermutung, strafrechtlich nicht relevante Fallkonstellationen bei der möglichen Veräußerung dieser Bilder unabhängig von der Frage ihrer Echtheit, die seit den inkriminierten Vorgängen inzwischen vergangene Zeit und der offene Umgang des Klägers mit dem gesamten Sachverhalt nicht berücksichtigt worden, sind ebenfalls nicht geeignet, ernstliche Zweifel an der von der Behörde und dem Verwaltungsgericht angestellten Gefahrenprognose zu begründen. Der Verweis auf die Unschuldsvermutung (s. Art. 6 Abs. 2 EMRK) verfängt schon deshalb nicht, weil die Unschuldsvermutung lediglich das strafprozessuale Verfahren und gerichtliche Entscheidungen betrifft, die an den Ausgang des Strafverfahrens anknüpfen, aber keine Aussage über die Zulässigkeit von Maßnahmen der Gefahrenabwehr trifft, denen in aller Regel eine Prognose über die künftige Entwicklung anhand eines Wahrscheinlichkeitsmaßstabs zugrunde liegt (stRspr, vgl. z.B. BVerwG, B.v. 25.3.2019 - 6 B 163.18 - juris Rn. 9; BayVGH, B.v. 29.10.2014 - 10 ZB 14.1355 - juris Rn. 7 m.w.N.). Weiter hat der Beklagte dem Kläger zu Recht entgegengehalten, dass er ausweislich der Erkenntnisse in den strafrechtlichen Ermittlungsverfahren wiederholt versucht habe, die Fälschungen als Originale zu verkaufen, der möglicherweise eintretende Schaden beim Käufer bei angeblichen Gemälden von Künstlern mit Weltruhm erheblich sei, nicht jeder private Käufer vor einem Kauf die Echtheit durch einen renommierten Gutachter prüfen lasse und ein Original nicht von einer Fälschung unterscheiden könne und auch ein länger zurückliegender Kunstfälscherprozess einem Verkauf einer Fälschung an private Käufer nicht entgegenstehe. Im Übrigen zeigt das gesamte Verfahren, dass der Kläger gerade nicht "einsichtig" war und ist, weiterhin die "Echtheit" seiner Bilder/Grafiken behauptet und deren Verkauf nach wie vor anstrebt.

[16] 1.3. Bereits im Ansatz verfehlt ist vor diesem Hintergrund (s. 1.2.) der Einwand fehlender Verhältnismäßigkeit (Art. 4 PAG) der Sicherstellungsanordnung, weil es dem Kläger dadurch auch nicht einmal möglich sei, „die Bilder als vermeintliche Fälschung zu veräußern“. Bei der rechtlich nicht zu beanstandenden Gefahrenprognose der Behörde und des Verwaltungsgerichts wäre eine solche „bedingte Freigabe" der Bilder zur Abwehr der gegenwärtigen Gefahr offensichtlich ungeeignet und damit zweckwidrig.

[17] 1.4. Das Verwaltungsgericht hat zu Recht auch einen Herausgabeanspruch des Klägers nach Art. 28 Abs. 2 Satz 1 PAG (n.F.) wegen nachträglichen Wegfalls der Sicherstellungsvoraussetzungen verneint.
[18] Der klägerische Einwand, die Verwendung der Bilder für künftige Straftaten sei zum insoweit maßgeblichen Zeitpunkt der letzten mündlichen Verhandlung "erst recht fernliegend“, weil er (Kläger) inzwischen rechtskräftig strafrechtlich verurteilt sei, sich geständig und reuig gezeigt habe und vor diesem Hintergrund gar nicht in der Lage sei, künftig entsprechende Straftaten zu begehen, sondern die Bilder schlicht zurückerhalten möchte, greift ebenso wenig durch wie die fortgesetzte Behauptung, es sei auch jetzt noch weitestgehend ungeklärt, ob es sich um Fälschungen oder Originale handle. Denn das Verwaltungsgericht hat überzeugend dargelegt, dass es sich mit Ausnahme der beiden vom Kläger selbst gemalten Bilder (Asservatennummern 1.25 und 1.26) bei den sichergestellten Bildern/Grafiken, die nach Angaben des Klägers alle aus der gleichen Quelle (s. oben 1.2.) stammen, aufgrund vorliegender (negativer) Gutachten und sachverständiger Einschätzungen sowie nicht aussagefähiger "positiver Analysen" um Fälschungen handelt. Auch hat der Beklagte in seiner Antragserwiderung zutreffend darauf hingewiesen, dass im verwaltungsgerichtlichen Verfahren erneut zwei vom Kläger ausgewählte Gemälde sowie ein Rahmen eines Bildes wissenschaftlich begutachtet worden seien und sich trotz gegensätzlicher Beteuerungen des Klägers als eindeutige Fälschungen erwiesen hätten; zu keinem einzigen Gemälde liege ein Gutachten vor, das die Urheberschaft des vermeintlichen Künstlers bestätige. Vielmehr bestätigten die strafrechtlichen Ermittlungsergebnisse, die Ergebnisse der Telefonüberwachung von Gesprächen mit den Mitbeschuldigten, das Verschleiern der tatsächlichen Herkunft der Gemälde und die Ergebnisse zu allen bislang begutachteten oder untersuchten Gemälden die erfolgte Bewertung als Fälschungen. Weiter hat der Beklagte zu Recht darauf verwiesen, dass auch die Gemälde ohne Signatur täuschungsgeeignet seien und im Übrigen die - von einem Mitangeklagten des Klägers praktizierte - Möglichkeit bestehe, dem Gemälde eine solche Signatur nachträglich anzufügen. Nicht zuletzt die vom Beklagten im Nachgang zur Antragserwiderung vorgelegten Informationen über zwei gegen den Kläger geführte erneute strafrechtliche Ermittlungsverfahren wegen versuchten gewerbsmäßigen Betrugs bzw. versuchten Betrugs im Zusammenhang mit dem Verkauf gefälschter Bilder (E-Mails des bayerischen Landeskriminalamts vom 22.7.2020 und 27.10.2020 mit Anlage, BI. 62/63 der VGH-Akte) belegen, dass die diesbezüglichen Einlassungen des Klägers nicht glaubhaft sind.

[19] Dass eine Markierung der sichergestellten Gemälde/Grafiken mit optischen Aufhellern oder fluoreszierenden Mitteln nicht geeignet ist, die fortbestehende Gefahr weiterer Betrugsstraftaten durch den Kläger im Zusammenhang mit dem Verkauf gefälschter Bilder effektiv zu beseitigen und demzufolge nunmehr einen Herausgabeanspruch zu begründen, haben sowohl das Verwaltungsgericht in der angegriffenen Entscheidung als auch der Beklagte in seiner Antragserwiderung überzeugend dargelegt. 
[20] 2. Die vom Kläger daneben geltend gemachten besonderen tatsächlichen und rechtlichen Schwierigkeiten der Rechtssache (§ 124 Abs. 2 Nr. 2 VwGO) sind im Zulassungsantrag weder hinreichend dargelegt worden, noch liegen sie vor.

[21] Der Kläger hat - wie oben ausgeführt - mit seiner Zulassungsbegründung nicht hinreichend dargelegt, welche begründeten Zweifel gegen die verwaltungsgerichtliche Entscheidung in tatsächlicher oder rechtlicher Hinsicht bestehen, die den Ausgang des Rechtsstreits als zumindest offen erscheinen lassen (vgl. Seibert in Sodan/Ziekow, Verwaltungsgerichtsordnung, 5. Aufl. 2018, $\S 124 a$ Rn. 209 und § 124 Rn. 106 ff.; Happ in Eyermann, VwGO, 15. Aufl. 2019, § 124a Rn. 68 und § 124 Rn. 27). Er hat auch nicht ausreichend verdeutlicht, weshalb der vom Verwaltungsgericht ermittelte Sachverhalt besonders unübersichtlich und die Sachverhaltsermittlung des Erstgerichts nicht ausreichend sein soll (vgl. Happ, a.a.O., § 124a Rn. 71). Der Hinweis allein auf den „umfangreichen Prozessstoff und die lange Prozessdauer" genügt den Darlegungsanforderungen jedenfalls nicht.
[22] Wie sich aus den obigen Ausführungen (unter 1.) ergibt, weist der vorliegende Fall weder von den maßgeblichen rechtlichen Grundlagen noch der anzustellenden Gefahrenprognose (gegenwärtige Gefahr i.S.v. Art. 25 PAG a.F.) her komplexe oder noch nicht geklärte Rechtsfragen auf.

[23] Eine ausführliche und auf alle aufgeworfenen Einzelfragen bzw. Einwendungen eingehende Begründung des angefochtenen Urteils allein ist noch kein entscheidendes Indiz für das Vorliegen besonderer Schwierigkeiten der Streitsache.

[24] Die Kostenentscheidung folgt jeweils aus § 154 Abs. 2 VwGO.

[25] Die Streitwertfestsetzung für die Zulassungsverfahren beruht auf $\S 63$ Abs. 2 Satz 1, § 47 Abs. 1 und 3 und $\S 52$ Abs. 1 GKG.

[26] Dieser Beschluss ist unanfechtbar ( $\$ 152$ Abs. 1 VwGO). Mit der Ablehnung des Antrags auf Zulassung der Berufung wird die Entscheidung des Verwaltungsgerichts rechtskräftig (§ 124a Abs. 5 Satz 4 VwGO).

\section{Streit um Sitz im Stiftungsrat des „Fürst-Pückler-Museum Park und Schloss Branitz"}

VerfGBbg, Beschluss vom 16. April 2021 - VfGBbg 71/18

\section{Tenor}

$\S 7$ Abs. 1 Satz 2 des Gesetzes über die Errichtung der Stiftung „Fürst-Pückler-Museum Park und Schloss Branitz" (SFPMG) vom 14. Dezember 2017 (GVBII Nr. 31) verletzt den Beschwerdeführer in seinem Grundrecht auf Gleichbehandlung aus Art. 12 Abs. 1 Satz 1 und Satz 2 Verfassung des Landes Brandenburg und ist nichtig.

Im Übrigen wird die Verfassungsbeschwerde teils verworfen, teils als unbegründet zurückgewiesen.

Das Land Brandenburg hat dem Beschwerdeführer seine notwendigen Auslagen für das Verfassungsbeschwerdeverfahren zu erstatten.

\section{Gründe}

\section{A}

[1] Der Beschwerdeführer wendet sich gegen eine gesetzliche Regelung zur Beteiligung der Erben des Fürsten Pückler im Stiftungsrat der Stiftung „Fürst-Pückler-Museum Park und Schloss Branitz”.
I.

[2] Der Beschwerdeführer ist zu einem Anteil von einem Zwölftel Mitglied einer ursprünglich aus sieben Personen bestehenden Erbengemeinschaft nach Theodora Gräfin von Pückler, geb. Gräfin zu Limburg-Styrum, die ihr Alleinerbe an Schloss und Park Branitz auf den 1871 kinderlos verstorbenen Hermann Ludwig Heinrich Fürst von Pückler-Muskau zurückführte und die im Zuge der Bodenreform 1946 auf besatzungsrechtlicher Grundlage enteignet wurde.

[3] Der Erbengemeinschaft nach Theodora Gräfin von Pückler wurden mit Bescheid des Landesamts zur Regelung offener Vermögensfragen vom 30. Oktober 2008 konkret bezeichnete 1.746 bewegliche Vermögensgegenstände zurückübertragen. Der Bescheid stellte den unentgeltlichen öffentlichen Nießbrauch der Stadt Cottbus an den Vermögensgegenständen gemäß $\S 5$ Abs. 2 Satz 1 Ausgleichsleistungsgesetz (AusglLeistG) bis zum 1. Dezember 2014 fest. Mit Bescheid des Landesamts zur Regelung offener Vermögensfragen vom 24. September 2014 wurden der Erbengemeinschaft nach Theodora Gräfin von Pückler konkret bezeichnete 4.673 bewegliche Vermögensgegenstände der Pückler-Callenberg-Bibliothek Branitz, die in die Landesbibliothek Potsdam überführt worden waren, zurückübertragen und der unentgeltliche öffentliche Nießbrauch gemäß § 5 Abs. 2 Satz 1 AusglLeistG bis zum 1. Dezember 2014 festgestellt. 